\title{
Dataset Reduction for Neural Network Based Digital Predistorters under Strong Nonlinearities
}

\author{
David López-Bueno \\ PHYCOM Department, CTD Division \\ Centre Tecnologic de Telecomunicacions de \\ Catalunya (CTTC/CERCA) \\ Castelldefels (Barcelona), Spain \\ david.lopez@,cttc.es
}

\author{
Pere L. Gilabert \\ Department of Signal Theory and \\ Communications, \\ Universitat Politècnica de Catalunya (UPC) \\ Castelldefels (Barcelona), Spain \\ plgilabert@tsc.upc.edu
}

\author{
Gabriel Montoro \\ Department of Signal Theory and \\ Communications \\ Universitat Politècnica de Catalunya (UPC) \\ Castelldefels (Barcelona), Spain \\ gabriel.montoro@upc.edu
}

\begin{abstract}
The artificial neural networks (ANN) are gaining momentum in the digital predistorters (DPD) thanks to their inherently good approximation capabilities. Under strong or complex power amplifier nonlinearities, the size of the ANN can increase and lead to long training periods which are unaffordable in fast-changing waveform scenarios like those proposed for $5 \mathrm{G}$ or 6G. In this work we combine the orthogonal matching pursuit technique together with dataset length reduction methods, to significantly shorten the ANN DPD coefficients update time.
\end{abstract}

Keywords - power amplifiers, predistortion, iterative learning control, machine learning algorithms, artificial neural networks

\section{INTRODUCTION}

Thanks to the digital predistorter (DPD) the power amplifier (PA) can be operated more efficiently when handling wideband high peak-to-average power ratio (PAPR), and meet the error vector magnitude or the adjacent channel power ratio (ACPR) requirements. Adaptive DPDs are nowadays needed to deal with changing environmental conditions and waveform scenarios and their complexity is increasing with the deployment of massive antenna transmitters. When modeling strongly nonlinear systems, the ANN DPD can offer global approximation capabilities in contrast to the inherent local approximating properties of the polynomial-based DPD. However, when the complexity of the ANN DPD is increased to model complex nonlinear phenomena, and requires large input datasets, the training time is severely increased. There is not a universal procedure to set up the best ANN for a given application but several works found in literature provide indications on the suitable architecture, activation functions and backpropagation algorithms or hyperparameter configurations [1]. Such works mainly refer to the indirect learning (IL) architecture shown in Fig.1 (left) that models the inverse response of the PA during the NN training and replicates the coefficients calculated in the forward path (NN inference). In this work, we target the input dataset reduction in the direct learning architecture (DL) shown in Fig. 1 (right), which models the counteracting distortion signal to be added to the original input signal to compensate for the PA distortion.

\section{Neural Network Adaptive Digital Predistorter}

In this work, we consider the real-valued time delayed feedforward NN in Fig. 2. According to the DL scheme, the input IQ pair is defined as the real and imaginary part of the NN input vector $u[n]$, and the output IQ pair will be an estimation of the real part and imaginary part of the $\mathrm{NN}$ expected output

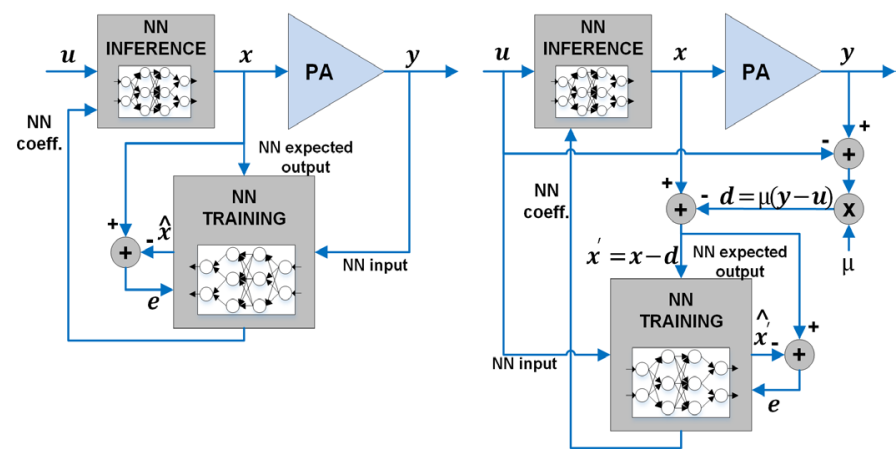

Fig. 1. ANN-based DPD IL (left) and DL (right) architectures.

$x^{\prime}[n]$. The input IQ pair is further processed by a group of data functions (DF in Fig. 2) to provide richer basis to the $\mathrm{NN}$ and improve the nonlinear modeling. The ANN DPD can benefit from the injection of envelope dependent terms (i.e. $\mid I_{i n}+$ $\left.j Q_{i n}\right|^{k}$ with $k \in \mathbb{N}$ ) [2], angle dependent terms (i.e. $\left(\operatorname{atan} 2\left(Q_{i n} / I_{i n}\right)^{k}\right.$ with $\left.k \in \mathbb{N}\right)$, or long term memory effects modeling components like those described in [3]. To enable dynamic nonlinear system identification, a linear time invariant (LTI) system built with tapped delay lines is added. In Fig 2. we have $N$ input dataset basis functions resulting from applying the LTI system to the IQ input signals and to the DF outputs. The input dataset length relies on the length of the IQ data being considered for modeling. The $\mathrm{NN}$ features one input layer (with $N$ inputs), two hidden layers with $F$ and $S$ neurons, and an output layer with 2 neurons. The neurons in the hidden layers employ the $\vartheta^{1}(\cdot)$ and $\vartheta^{2}(\cdot)$ nonlinear activation functions and a pure linear activation function is used for the output layer. This NN architecture has $N F+F S+2 S$ weights and $F+S+2$ biases. In Fig. $2, w_{i j}^{k}$ is the $j^{\text {th }}$ weight belonging to the $i^{t h}$ neuron in the $k^{\text {th }}$ layer. Similarly, $\theta_{i}^{k}$ is the bias belonging to the $i^{t h}$ neuron in the $k^{t h}$ layer. In the forward pass we have that

$$
\begin{aligned}
& \hat{I}_{\text {out }}[n]= \\
& \left.=\sum_{k=1}^{S} w_{1 k}^{3} \vartheta^{2}\left(\sum_{j=1}^{F} w_{k j}^{2} \vartheta^{1}\left(\sum_{i=0}^{N} w_{j i}^{1} \varphi_{i}[n]+\theta_{j}^{1}\right)+\theta_{k}^{2}\right)+\theta_{1}^{3}\right) \\
& \hat{Q}_{\text {out }}[n]= \\
& \left.=\sum_{k=1}^{S} w_{2 k}^{3} \vartheta^{2}\left(\sum_{j=1}^{F} w_{k j}^{2} \vartheta^{1}\left(\sum_{i=0}^{N} w_{j i}^{1} \varphi_{i}[n]+\theta_{j}^{1}\right)+\theta_{k}^{2}\right)+\theta_{2}^{3}\right)
\end{aligned}
$$




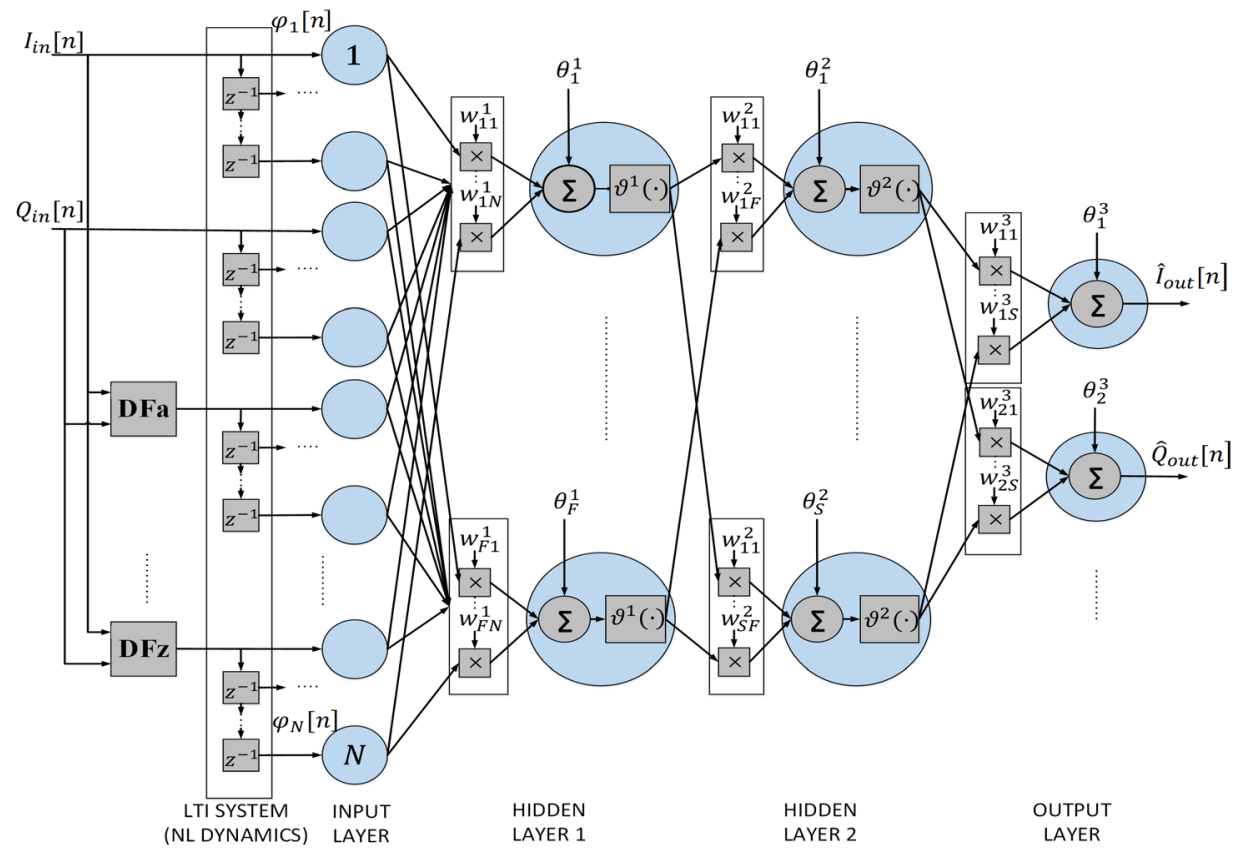

Fig. 2. Two-hidden layer real-valued feedforward shallow ANN architecture.

The Levenberg-Marquardt (LM) backpropagation algorithm is used in our ANN to calculate the NN coefficients by minimizing the cost function $E$, where

$$
\begin{aligned}
E=\frac{1}{2 K} \sum_{n=1}^{K}\left[\left(I_{\text {out }}[\right.\right. & {\left.[n]-\hat{I}_{\text {out }}[n]\right)^{2} } \\
& \left.+\left(Q_{\text {out }}[n]-\hat{Q}_{\text {out }}[n]\right)^{2}\right] \\
& =\frac{1}{2 K} \sum_{n=1}^{K}|\boldsymbol{e}|^{2},
\end{aligned}
$$

for every input data batch of length $K$. This forward-backward process is repeated until the desired modeling performance is achieved or the $\mathrm{NN}$ fails in generalization.

When going backward, the LM modification to the GaussNewton method [4] minimizes $E$ with respect to a parameter $\boldsymbol{c}$ with the weights and biases, which is updated as

$$
\boldsymbol{c}^{n+1}=\boldsymbol{c}^{n}-\left[\boldsymbol{J}^{T} \boldsymbol{J}+\beta \boldsymbol{I}\right]^{-1} \boldsymbol{J}^{T} \boldsymbol{e}
$$

where

$$
\boldsymbol{c}=\left[w_{11}^{1} \ldots w_{F N}^{1} \theta_{1}^{1} \ldots \theta_{F}^{1} \ldots \ldots w_{11}^{3} \ldots w_{2 S}^{3} \theta_{1}^{3} \theta_{2}^{3}\right],
$$

where $\boldsymbol{I}$ is the identity matrix, $\beta$ is a learning rate parameter and $\boldsymbol{J}$ is the Jacobian matrix calculated over the error vector $\boldsymbol{e}$ with respect to $\boldsymbol{c}$ as

$$
\boldsymbol{J}=\left[\begin{array}{ccccc}
\frac{\partial e[1]}{\partial w_{11}^{1}} & \frac{\partial e[1]}{\partial w_{12}^{1}} & \cdots & \frac{\partial e[1]}{\partial \theta_{1}^{3}} & \frac{\partial e[1]}{\partial \theta_{2}^{3}} \\
\frac{\partial e[2]}{\partial w_{11}^{1}} & \frac{\partial e[2]}{\partial w_{12}^{1}} & \cdots & \frac{\partial e[2]}{\partial \theta_{1}^{3}} & \frac{\partial e[2]}{\partial \theta_{2}^{3}} \\
\vdots & \vdots & \vdots & \vdots & \vdots \\
\frac{\partial e[K]}{\partial w_{11}^{1}} & \frac{\partial e[K]}{\partial w_{12}^{1}} & \cdots & \frac{\partial e[K]}{\partial \theta_{1}^{3}} & \frac{\partial e[K]}{\partial \theta_{2}^{3}}
\end{array}\right] .
$$

\section{DATASET REDUCtion}

In our ANN, we define the $L x N$ input dataset matrix $\boldsymbol{U}=$ $(\varphi[1], \ldots, \varphi[n], \ldots, \varphi[L])$ with $n=1, \ldots, L$ and $\boldsymbol{\varphi}^{T}[n]=$ $\left(\varphi_{1}[n], \ldots \varphi_{i}[n], \ldots, \varphi_{N}[n]\right)$ with $i=1, \ldots, N$. We will reduce the two $\boldsymbol{U}$ matrix dimensions. By reducing the number of basis $(N$ in Fig. 2) both the number of coefficients at the first hidden layer and the training time are reduced. The latter will be further impacted by applying dataset length reduction techniques.

\section{A. Dataset basis reduction}

The Orthogonal matching pursuit (OMP) technique can be used as an a priori offline study to determine the best basis functions to linearize the PA. This process is applied once and the basis selection will be kept for a given scenario (i.e. fixed PA output power, waveform and bandwidth). The support vector containing the indices of the basis functions in $\boldsymbol{U}$ is defined as $\boldsymbol{P}^{(m)}$ where $m_{\max }=N$ is the maximum number of basis under study. As shown in the procedure of Algorithm 1,

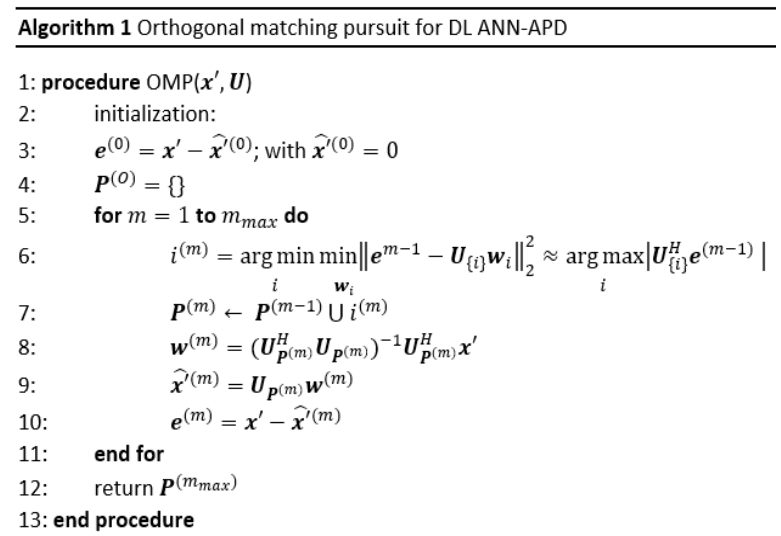


at every iteration of the OMP search, $\boldsymbol{P}^{(m)}$ is fed with the indices that better contribute to minimize the residual modeling error and are therefore sorted according to their relevance. A Bayesian information criterion (BIC) can be used to determine the optimum number of basis and the support set can be truncated for a given prunning factor (i.e. $\left.\boldsymbol{U}_{\left.\boldsymbol{P}^{\left(1: m_{\max }\right.} / \text { pfactor }\right)}\right)$. In [5], the authors propose the doubly OMP (DOMP) scheme that adds a Gram-Schmidt orthogonalization step into the basis selection procedure which contributes to better normalized mean square error (NMSE) performance.

\section{B. Dataset length reduction}

Several sample selection methods (SSM) have been reported in literature [6] to reduce the computational complexity of DPDs. In this work, we introduce a data batch selection (BS) method. The BS method takes the relevant waveform data batches in $\boldsymbol{u}$ depending on the characteristics of the PA output $\boldsymbol{y}$. Two variants of the BS method are presented: consecutive BS (CBS) and sparse BS (SBS). The procedure has the following steps: 1) $\boldsymbol{u}$ and $\boldsymbol{y}$ are divided into a number of data batches $N_{b}$ which is an integer multiple number of the waveform length reduction factor $R$ to be applied. The number of batches to be selected is $N_{s}=N_{b} / R$. 2) With CBS, a sliding window of consecutive samples with length $L_{s}=N_{s} L / N_{b}$ is shifted over $\boldsymbol{u}$ and $\boldsymbol{y}$, with $L / N_{b}$ shifting step, to calculate both the NMSE, the adjacent channel error power ratio (ACEPR) and the $\boldsymbol{u}$ signal mean power. When using SBS, all the metrics are calculated individually for each of the $N_{b}$ batches (there is not a wider sliding window). 3) The BS algorithm is in charge of sorting the expanded batch indices (CBS) or the sparse batch indices (SBS) in order of importance according to featuring simultaneously the worst NMSE and ACEPR values, and the highest mean power to avoid the impact of the noise in the nonlinear modelling. The reduced NN input waveform will be thus populated with either a consecutive portion of $\mathrm{u}$ in $\mathrm{CBS}$ or a sparse number of portions in SBS, as shown in Fig. 3, that meet the previous criterion.

In the next section, the BS methods are compared to the histogram-based SSM mesh selecting (MeS) which is an evolved version of the work in [7] with multidimensional IQ mapping to better account for wideband PA memory effects.

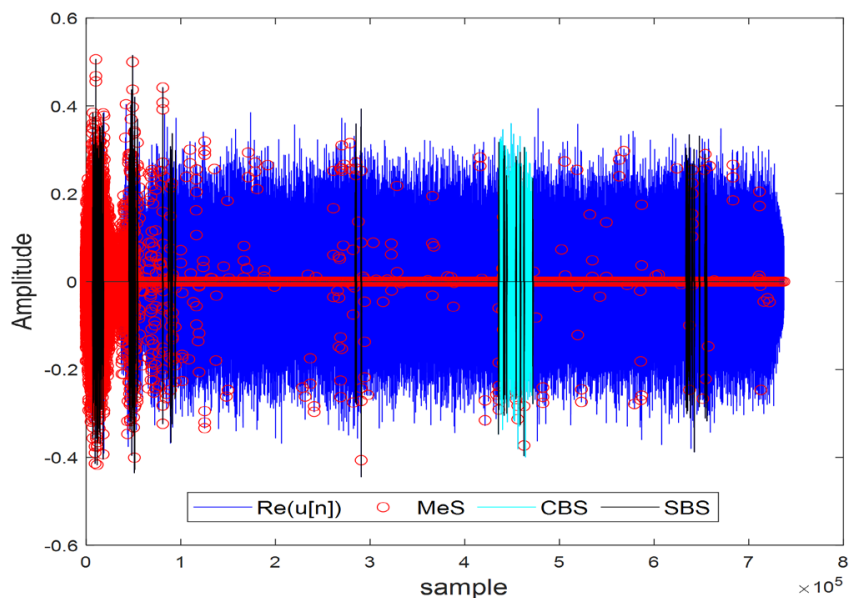

Fig. 3. MeS, CBS and SBS input dataset length reduction methods (i.e. for 20reduction factor).

\section{EXPERIMENTAL TEST SETUP AND RESULTS}

The dataset reduction techniques have been benchmarked and validated with the Matlab controlled hardware test bench shown in Fig. 4. A GaN HEMT class J PA which is operated at $875 \mathrm{MHz}$ RF frequency and $28 \mathrm{dBm}$ mean output power with $80 \mathrm{MHz}$ bandwidth signals featuring 13-14 dB PAPR. The DPD neural network has been implemented in Matlab. The dataset has 105 inputs/basis each with 737280 samples. These 105 inputs belong to the I and Q components and the augmented products until the sixth power, considering up to 12 consecutive delays, and a single long term memory effects modeling component averaging the IQ input signal power over a finite window. The hidden layers have 20 neurons each and use hyperbolic tangent sigmoid activation functions.

The OMP and the length reduction techniques are evaluated independently in Fig. 5 and Fig. 6. The combined use is validated in Table I. Both in Fig. 5 and Table I the reduction factors being considered are displayed after the dataset reduction technique acronyms. Fig. 5 shows how the complex ANN DPD performs better than the generalized memory polynomial (GMP) DPD when using the DPD coefficients that provide the maximum (MAX) performance. The ANN DOMP is better than the ANN OMP in 1-1.5 dB of NMSE but features similar ACPR, when using a reduction factor of 8. Using ANN DOMP with this reduction factor also outperforms the GMP DPD. In Fig. 6, for lower reduction factors CBS is a good choice while in large reduction factors SBS is the best choice. The plot shows that even considering a reduction factor of 320 the NN still fulfills reasonable NMSE and ACPR. Finally, Table I shows how combining the two types of dataset reduction techniques can be beneficial. Combining basis reduction and length reduction in the NN implies an equivalent reduction both in terms of coefficients and training time (measured with Matlab's tictoc and involving 8 DPD iterations), and better performance than only using the best BS dataset length reduction method (i.e. see SBS160 vs CBS40+DOMP4 in Table I). These results indicate that by using the input dataset reduction techniques the NN training time can be dramatically reduced and potentially be in the order of magnitude of the classical polynomial DPD adaptation time.
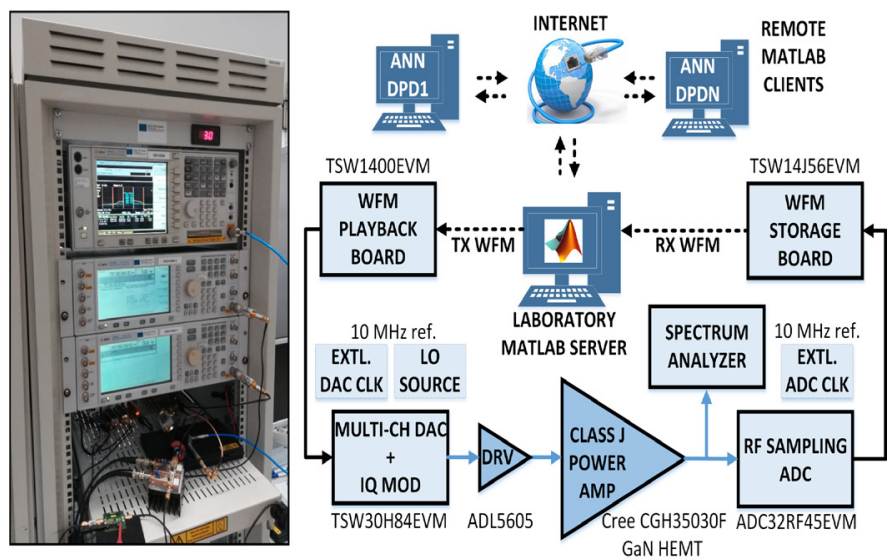

Fig. 4. Remotely accessible digital linearization test setup. 

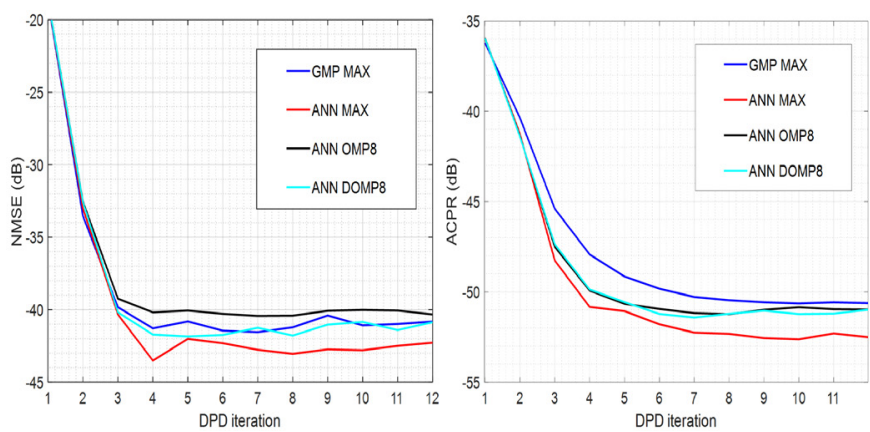

Fig. 5. NMSE and ACPR vs DPD iteration under dataset basis reduction.

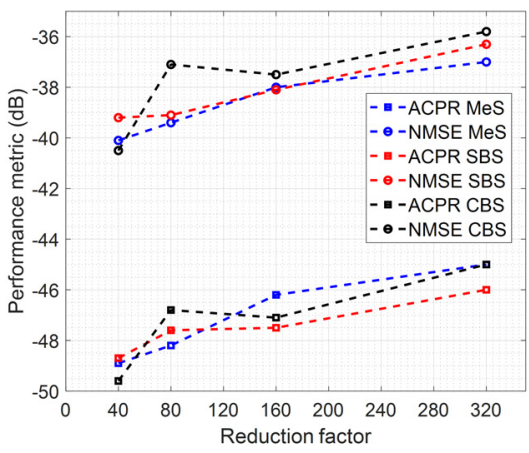

Fig. 6. NMSE and ACPR vs dataset length reduction factor and method.

TABle I. COMPleXity, Timing AND Performance Benchmark

\begin{tabular}{|l|c|c|c|c|}
\hline \multirow{2}{*}{ Method } & \multicolumn{4}{|c|}{ Performance Benchmark } \\
\cline { 2 - 5 } & $\begin{array}{c}\text { DPD } \\
\text { coeffs. }\end{array}$ & $\begin{array}{c}\text { 8-Iteration DPD } \\
\text { Update Time (s) }\end{array}$ & $\begin{array}{c}\text { NMSE } \\
(\boldsymbol{d B})\end{array}$ & $\begin{array}{c}\boldsymbol{A C P R} \\
(\boldsymbol{d B})\end{array}$ \\
\hline NN MAX & 2582 & $8 \mathrm{e} 4$ & -42.6 & -52.5 \\
\hline NN DOMP4 & 1002 & $25 \mathrm{e} 3$ & -41.4 & -51.4 \\
\hline NN CBS40 & 2582 & 660 & -40.5 & -49.6 \\
\hline NN SBS160 & 2582 & 230 & -38.1 & -47.5 \\
\hline NN CBS40+DOMP4 & 1002 & 104 & -39.2 & -48.2 \\
\hline GMP MAX & 322 & 320 & -40.7 & -50.6 \\
\hline
\end{tabular}

\section{CONCLUSION}

The direct learning ANN DPD can outperform the classical DPD schemes at the cost of a potentially unaffordable training time for adaptive scenarios. OMP combined with dataset length reduction strategies is effective for selection of $\mathrm{NN}$ inputs, $\mathrm{NN}$ coefficient reduction, and to greatly cut the $\mathrm{NN}$ training time for DPD adaptation while fulfilling signal quality requirements. Application to MIMO DPD schemes and further work on joint design of dataset length reduction methods and feature selection techniques needs to be conducted to reach the best trade-off between NN performance and the DPD update time.

\section{ACKNOWLEDGMENT}

The authors would like to thank Dr J.A. García, Dr M.N. Ruiz, Dr J.R. Pérez Cisneros, and D. Vegas from the Universidad de Cantabria for the donation of the class-J PA. This work was supported in part by the Spanish Government (Ministerio de Ciencia, Innovación y Universidades) and the Fondo Europeo de Desarrollo Regional (FEDER) under grants RTI2018-099841-B-I00 and TEC2017-83343-C4-2-R, and in part by the Generalitat de Catalunya under grants 2017 SGR 891 and 2017 SGR 813.

\section{REFERENCES}

[1] M. Rawat, K. Rawat and F. M. Ghannouchi, "Adaptive Digital Predistortion of Wireless Power Amplifiers/Transmitters Using Dynamic Real-Valued Focused Time-Delay Line Neural Networks," in IEEE Trans. Microw. Theory Techn., vol. 58, no. 1, pp. 95-104, Jan. 2010.

[2] D. Wang, M. Aziz, M. Helaoui and F. M. Ghannouchi, "Augmented RealValued Time-Delay Neural Network for Compensation of Distortions and Impairments in Wireless Transmitters," in IEEE Trans. Neural Netw. Learn. Syst., vol. 30, no. 1, pp. 242-254, Jan. 2019.

[3] A. S. Tehrani, T. Eriksson and C. Fager, "Modeling of long term memory effects in RF power amplifiers with dynamic parameters," 2012 IEEE/MTT-S International Microwave Symposium Digest, Montreal, QC, 2012, pp. 1-3.

[4] M. T. Hagan and M. B. Menhaj, "Training feedforward networks with the Marquardt algorithm," in IEEE Transactions on Neural Networks, vol. 5, no. 6, pp. 989-993, Nov. 1994

[5] J. A. Becerra et al., "A Doubly Orthogonal Matching Pursuit Algorithm for Sparse Predistortion of Power Amplifiers," in IEEE Microw. and Wireless Comp. Letters, vol. 28, no. 8, pp. 726-728, Aug. 2018.

[6] J. Kral et al., "On Feedback Sample Selection Methods Allowing Lightweight Digital Predistorter Adaptation," in IEEE Trans. Circuits Syst. I, Reg. Papers, vol. 67, no. 6, pp. 1976-1988, Jun. 2020.

[7] T. Wang, P. L. Gilabert and G. Montoro, "Under-sampling effects and computational cost reduction in RF power amplifier behavioral modeling," 2015 10th European Microwave Integrated Circuits Conference (EuMIC), Paris, 2015, pp. 57-60. 